\title{
Improving nursing home falls management program by enhancing standard of care with collaborative care multi-interventional protocol focused on fall prevention
}

\author{
Karen M. Jackson * \\ School of Nursing, Loyola University New Orleans, USA
}

Received: December 9, 2015

Accepted: February 22, $2016 \quad$ Online Published: April 11, 2016

DOI: $10.5430 /$ jnep.v6n6p84

URL: http://dx.doi.org/10.5430/jnep.v6n6p84

\begin{abstract}
Purpose: The objective of this quality assurance project was to implement a collaborative multi-strategy fall risk management program to reduce patient falls within a nursing home.

Design: A quasi-experimental study.

Intervention: A multi-interventional program based on recommendations from Agency for Healthcare Quality was instituted. Direct care providers were required to conduct every 2-hour rounding on patients. The staff was required to participate in organizational education and training on fall prevention strategies. Patients were encouraged to participate in activities outside of their rooms throughout the day. Monthly meetings were held to review fall occurrences, collaborate on project initiatives, and discuss trends in fall rates.

Setting and Sample: The setting for this project was a 150-bed nursing home in suburban Texas. A total of 10 participants were recruited for the fall team, and interventions were performed on each of three nursing units. The median age of the patient population was 75 years old of which $46 \%$ are males, and $53 \%$ are females.

Measurement: The Falls Management Program-How to Reduce Fall questionnaire was completed before and after the intervention period to evaluate participants' knowledge about falls and prevention. Quality assurance data were reviewed, analysis of documentation for rounding, activities, and falls data was completed.

Results: The pre-implementation mean fall rate per month was 24.5 (average monthly census was 120 patients), compared to 2-month and 4-month post-implementation mean rates which were 13.5 (average monthly census was 116 patients), and 9.5 (average monthly census was 111 patients). A knowledge test "How to Reduce Falls" was administered to the fall team. Average scores were proportionately better on the 4-month Post-test (2-month Post-test: $p=.037$ ), and (4-month Post-test: $p=.029$ ). Additionally, statistical results indicate there is a significant difference in falls rate comparing pre-implementation to 4-month post-implementation $(t(2)=25.981, p<.05)$.

Discussion: Implementation of best quality fall prevention programs can improve the overall health and quality of life of patients. Care providers must be vigilant in consistently facilitating evidence-based practices that contribute to best outcomes for every patient. In so doing, enhanced standards of care will circumvent patient falls and promote best patient outcomes.
\end{abstract}

Key Words: Falls, Elderly falls, Nursing home care, Fall management, Hourly rounds

${ }^{*}$ Correspondence: Karen M. Jackson; Email: kmjackson@ @oustonmethodist.org; Address: School of Nursing, Loyola University New Orleans, USA. 


\section{INTRODUCTION}

Falls and fall-related injuries in the elderly are universally prevalent, and the ageing populace is vulnerable to its effects both personally and economically. ${ }^{[1]}$ More importantly, elderly falls is the fifth leading cause of death by fall-induced injuries, and is attributed to being the most common cause of longstanding pain and suffering in this population. According to the Centers for Disease Control and Prevention (CDC), ${ }^{[2]} 40$ million people age 65 and over lived in the United States in 2010, accounting for 13 percent of the total population. Moreover, nearly 1,703,398 elderly residents occupied beds in certified nursing home facilities. Because innovative advances in technology and healthcare are prolonging the lives of the general population, healthcare clinicians must promote a culture that supports evidence based fall prevention interventions which reduce risk and prevalence of patient falls in LTC facilities.

Studies that have been conducted on fall prevention which have been effective in clinical practice include a study conducted by Rapp et al. (2008) in which a secondary analysis of a cluster randomized control trial (RCT) evaluated the effectiveness of a multifactorial fall prevention program in pre-specified sub-groups of nursing home residents. ${ }^{[3]}$ It included 725 participants wherein variables studied were staff and resident education, environment change, hip protectors, and patient exercise. Statistical test incorporated the Wald Test to measure results. Results revealed interventions were more effective in participants with cognitive impairment, prior fall history, urinary incontinence, and with no mood problems. Weakness of the study may have included underreporting of falls in intervention group and control group, and systematic underreporting in specific subgroups. Validity and reliability were recognized, and findings indicated that allocation of resources may be of relevance in fall prevention, especially underreporting of subgroups.

Another study was a randomized control trial conducted by Sherrington et al. (2008) to evaluate the effectiveness of a multifactorial intervention on incidence of alls in psychogeriatric nursing home patients. ${ }^{[4]}$ The study included 518 participants. There were several variables considered which included fall risks, assessments, fall history, medication intake, mobility, assistive devices, team engagement, patient length of stay, and fall prevention. Researchers used multilevel poisson regression analysis to measure results. Key findings illustrated introduction of a structured multifactorial intervention to prevent patient falls can significantly reduce falls in the nursing home patients. Weaknesses include concern that unintentional selection of highly motivated nursing homes was selected, reasons for dropout were not registered, and the potential for selection bias was noted.
A meta-analysis study was conducted to examine the effectiveness of interventions used for decreasing the fear of falling by investigating individual studies on the prevention of fear of falls. ${ }^{[5]}$ It included 957 participants. Variables studied were exercise, education and hip protectors. Statistical tests used included the variability of effect using Q statistic. Upon completion of the study, the findings indicated a positive correlation regarding combining exercise and education effectively reduced fear of falling. Results of this study emphasizes the necessity of empowering caregivers to combine activities such as exercise, education, encouragement and socialization to effectively decrease elderly patients fear of falling.

Another study involved a longitudinal quasi-experimental design with the aim of evaluating the effects of a training curriculum based on TeamSTEPPS with video vignettes focusing on fall prevention for debriefing and reinforcement on team members' safety was completed. ${ }^{[6]}$ There were 34 participants included in this study. Variables included staff training "TeamSTEPPS", and encouraging a safety culture. Statistical tests included frequencies, $x 2$ tests, repeated measures, analysis of variance, $t$-Tests, and Chi-square tests. Upon completion of the study, the results revealed combined team training fall prevention interventions with improved communication between interdisciplinary members related to patient's fall risks status was effective for reducing falls. Weaknesses included lack of consistent interdisciplinary group participation during training.

\subsection{Background and significance}

One of the most important safety challenges that exist for care providers is patient falls. Amongst the elderly, falls have become the most common adverse event (AE) in healthcare facilities. ${ }^{[7]}$ While evidence has shown that many falls have not resulted in death or serious physical injury, in comparison with community-dwelling elderly persons, falls in institutions have the propensity for effecting more serious complications, with $10 \%-25 \%$ resulting in fracture or laceration. ${ }^{[8]}$ According to the CDC, annually there are at least 2 million elderly who require treatment in emergency departments with fall-related injuries, and national statistics indicate falls to be the leading cause of deaths due to injury in adults who are over 65 years in the United States, accounting for more than 10,000 deaths annually. ${ }^{[9]}$

Despite the ongoing debate of what constitutes a fall, the National Database of Nursing Quality Indicators (NDNQI) defines a patient fall as an unplanned descent to the floor with or without injury to the patient. ${ }^{[10]}$ Thus, the CDC has found the occurrence of patient falls in a typical 100-bed nursing home (NH) averages 100 to 200 per annum, which justifies 
the need for instituting best practices in patient safety and fall prevention. Injurious falls have been linked to negative outcomes such as hip fractures, head and brain injuries, and broken limbs that can cause several complications which limit independence and lower the quality of a person's life. ${ }^{[11]}$

Understanding and clarifying assumptions related to elderly falls by use of evidence-based clinical practices are fundamental in improving patient safety and quality of care. Hill et al. indicates the etiology of falls may be a combination of both intrinsic (e.g., existing co-morbidity) and extrinsic (e.g., environmental) risk factors, which generally present a difficult clinical problem when treating older adults. ${ }^{[12]}$ From Hill and colleagues' perspective, falls incite sequelae that include worsened morbidity and mortality, increased health care system costs and physical as well as psychosocial consequences. Distinguishing precursors to patient falls include cognitive impairment, generalized weakness and unsteady gait, environmental factors, inappropriate footwear, medications, inadequate staffing, and lack of effective fall management protocols. Furthermore, researchers have found that fear of falling is an independent predictor of falls and functional decline, which has a negative impact on older adults in all settings including nursing. ${ }^{[13]}$

In terms of patient falls and fall-related injuries (FRIs), adverse consequences associated with falls are associated with increased morbidity, mortality, and the unfavorable impact on human health status, health system services and medical cost. ${ }^{[14]}$ Most common adverse consequences of patient falls that affect patient outcomes include pain and suffering, hospitalization, the potential exacerbation of co-morbidities, delayed recovery, mistrust, and possibly a government investigation. In the literature, it has been noted that nursing home residents who are concerned about falls or fall-related injuries may avoid ambulation, reduce activity participation, and be less self-sufficient in personal care which induces further decline with independence. ${ }^{[15]}$ Costs of hospitalization and medical management related to falls and its related injuries in the elderly are estimated to exceed $\$ 43$ billion by 2020 , which has caused great concern because the incidence of falls with advanced age is more significant with each decade of life. ${ }^{[16]}$

In the literature, several studies have acknowledged the elderly as a vulnerable population with increased risks for falls; therefore a collaborative care multi-strategy fall prevention initiative would reduce falls among nursing home residents and improve clinical outcomes. Ideal nursing environments rely on hourly rounding as a systematic, proactive nursedriven evidence-based intervention that is integral in anticipating and addressing the needs of hospitalized patients. ${ }^{[17]}$
Moreover, experts have indicated that patient safety is a fundamental component of hourly rounding, as well as a priority impacting the care delivery process. One of the most effective methods used in fall prevention is every 1 to 2 hour rounding to assess patient's needs. ${ }^{[18]}$

\subsection{Framework}

The Patient-Centered Nursing Framework places high value on interwoven relationships between the care environment (structure measures), nurses' professional proficiencies (structure measures), caring process (process measures), and patient outcomes (outcome measures), especially for use of evaluating implementation of fall prevention programs and reducing fall rates. ${ }^{[19]}$ Modified from their original framework the authors integrated features of the caring perspective which is comprised of five approaches: understanding the best interests of patient's beliefs and values, providing for patients physical needs, having an empathetic presence, sharing decision making with patients and their families, and engaging patients and their families in fall prevention goals. ${ }^{[19]}$ The education goals for nurses and other primary care providers must promote their professional knowledge and skills in implementing required fall management programs, as well as cultivating their caring attitudes of treating patients like their own families - the principles of providing patient-centered and relationship-based care at the bedside. ${ }^{[20]}$

\subsection{Objective}

This was a quality assurance pilot project to enhance standard of care for a fall management program designed for adult patients in a long-term care setting in which falls were problematic. The objective of this project was to enhance standard of care by utilizing a collaborative multi-strategy fall risk management initiative to reduce patient falls in a long term care (LTC) setting. Approximately $30 \%$ to $50 \%$ of fall incidents and associated trauma can be reduced with initiation and implementation of timely, relevant and effective fall-prevention modalities. ${ }^{[21]}$ From a quality of care perspective, the coordination and implementation of evidence-based interventions that are aligned with a well-established theoretical framework will support best patient outcomes in fall management. This scholarly project involved formalizing a fall risk prevention program, based on the existent informal and formal patterns currently used within the quality assurance program at the institution to enhance effective outcomes.

The program included recommended evidenced-based multiparty parameters for falls prevention which resulted in development of new processes to improve clinical care and enhance patients overall care experience and quality of life. 
Interventions supporting McCormack and McCance's theoretical framework which undergirds the theoretical personcentered nursing model aligned by principles of patientcentered and relationship-based care were essential in this study. ${ }^{[19]}$

\subsection{Clinical question}

The clinical question is: will implementation of a multiintervention fall prevention project significantly reduce elderly patient falls in a nursing home, and if so, to what degree?

\section{METHODOLOGY}

\subsection{Design}

This project was based on a quality assurance/improvement model, reinforcing standard of care and supplementing with: (a) pre- and post-test evaluation for participant knowledge about falls and prevention, (b) added documentation for every 2-hour rounding component, increased patient activities components, (c) introduction of a formal falls team approach, and (d) expanded evaluation of effectiveness of the program overall in falls prevention (reduction of falls incidence). A quantitative design was used, and a convenience sample of direct care providers who met inclusion criteria participated as fall team members. Data were strategically collected and statistically analyzed to demonstrate the efficacy of project implementation. Existent protocols for standard of care were reinforced and supplemented during this project, with added education, extended monitoring and documentation.

\subsection{Setting}

The setting for data collection was at a 150-bed nursing home located in suburban Texas. The facility's average daily census was 123 , with median patient age 75 years old and gender of $46 \%$ male, and $53 \%$ female. There was a diverse patient population which included various ethnicities such as African Americans, Caucasians, Hispanics, Asians, and Middle Easterners. Excluding the Dementia Unit, at least 44\% of the patients utilized a wheelchair or rolling walker, and required minimal to moderate assistance with transfers. The other $56 \%$ of patients were non-ambulatory. The facility was committed to continually improving its practices which included learning, understanding, and meeting the needs of the population's diverse ethnic and multicultural backgrounds.

The convenience sample of participants in this project included 10 staff participants who were involved in direct patient care who made up the falls management team, in addition to leaders of the organization. Team participants were from various disciplines, which included one registered nurse manager, three licensed vocational nurses, one physi- cal therapist, one occupational therapist, one restorative care assistant, one medication aide, and two nurse care assistants. Inclusion criteria were that participants had to provide direct patient care, and be employed full-time for at least six months at the nursing home. Exclusions included any person working part-time, or considered a contract worker.

The fall team was recruited based on recommendations of AHRQ's evidence based-practices to include a multiinterventional program instituted by collaborative team members with a common focus on fall prevention is effective. ${ }^{[22]}$ Recruitment of participants occurred during introduction of program objectives at an initial luncheon meeting held with administrative leaders of the facility. Organizational leaders identified individuals with diverse roles and responsibilities as direct care providers based on inclusion and exclusion criteria, and invited them to the introductory meeting. Ten employees agreed to participate on the fall management team, and completed the pre-test for knowledge.

The fall team $(\mathrm{n}=10)$ was made up of the following: nurses $(n=4 ; 40 \% ; 1-R N$, and 3-LVNs), therapists ( $n=2 ; 20 \%$; 1-PT, and 1 -OT), and nurse aides ( $\mathrm{n}=4 ; 40 \%$; 1 -RCA, 1-MCA, and 2-PCAs). Team representatives were culturally diverse in this manner: Caucasian $(\mathrm{n}=1 ; 10 \%)$, African American $(\mathrm{n}=6 ; 60 \%)$, Hispanic $(\mathrm{n}=2 ; 20 \%)$, and Filipino $(\mathrm{n}=1$; $10 \%)$. The team was predominantly female $(\mathrm{n}=9 ; 90 \%)$. Lastly, ages of team members were in this fashion: 50 years and older $(n=4 ; 40 \%)$, forty to forty-nine years $(n=2 ; 20 \%)$, and thirty to thirty-nine years $(n=4 ; 40 \%)$. In general, direct caregivers at the long-term healthcare organization included nurse aides who completed their high school education and obtained a nurse aide certification (CNA), restorative care aides who were CNAs with additional training in providing exercise repetitions to patients, certified licensed vocational nurses, and physical therapy assistants who have completed courses and obtained a certification in physical therapy. And, equipment that was commonly used at the facility included balance bars, 2-pound and 5-pound hand weights, 2-pound ankle weights, hand pedal exercise machines, gait belts, tension exercise resistance bands, and adjustable staircases.

\subsection{Intervention}

\subsubsection{Mobility training}

The Director of Physical Therapy provided mobility training to the Falls Team and staff. Training consisted of assessment of potential environmental fall risk, safe mobility with transfers, gait training, and muscle strengthening and balance. Organization-wide coaching to reinforce compliance with wearing gait belts while on duty. All participants received instruction on the importance of patient education in falls prevention, participation in daily activities in the main activity 
room, as well as assurance of activities in plans of care.

\subsubsection{Q2 hour patient rounding}

Fall prevention strategies included every two hour rounding focused on the 4Ps (potty, pain, positioning, and possession), and documentation of rounding. Compliance with documentation on rounding tool was reinforced throughout implementation. Encouragement of routine activities for patients outside of their rooms (activity room) daily and throughout the day, and based on plan of care, physical therapy regimen if a patient has fallen, has increased risks (BRIGGS), or in recovery from a fall is the reason for admission. For those patients who are not involved in a regular physical therapy regimen, restorative care physical activities will be developed based on plans of care.

\subsubsection{Team meetings}

Monthly meetings were held with falls management team members. The PI coordinated meetings, and developed agenda items collaboratively with the director of physical therapy. Continued educational updates were provided to all staff throughout the facility to reinforce program initiatives, identify barriers and risks might prevent implementation and proposed strategies, and provided ongoing discussions related to project success. Two months after project implementation, staff participated in an organization-wide falls management training that was based on evidenced-based fall prevention programs. Training an organizational priority for ensuring staff understood their roles in patient safety, and their adherence to fall prevention initiatives.

The program included documentation, falls risk assessment, education, restorative or physical therapy (muscle strengthening and balance, safe training related to gait and transfers), and hourly staff rounding. "Rounding with Purpose" was a key intervention of the Falls Team to fall prevention. Direct care staff engaged patients to participate in activities outside of their rooms. For those patients who remained in their rooms due to physical immobility, a direct care staff member was assigned to complete every two hour rounding.

Following any fall, standard procedures of the institution were followed.

Per institutional protocol, the director of nursing (DON) recorded events from the Incident/Accident Report to the Incident/Accident Report QA\&A Log, and then met with the PI on a monthly basis to review. The PI monitored documentation of staff re-training and any in-services after identified fall events. Post fall, an order can be written for patients to be evaluated for further therapy services. Total project duration was approximately four months.

\subsection{Measurement tools}

Several monitoring tools were utilized to measure outcomes related to program strategies in reducing patient falls. A questionnaire was used to evaluate staff knowledge related to fall reduction. A fall risk assessment tool was utilized to validate documentation of fall risk assessment and scores on admission. Staff rounding notation sheets were monitored for documentation of assessment of the 4Ps (potty, pain, position and possession). Rounding sheets were checklist based and indicated date and hours for staff to check off identifying the 4Ps. Abstraction of quality assurance data included completing chart audits to validate completion of BRIGGS Fall Risk Assessment forms upon patient admission to the facility. Within two business days upon admission, nurses assessed patients for potential fall risk. Residents that obtained a total score of 10 or greater were considered as being at high risk for falls. Patients considered with risk of falling were recommended for further fall prevention strategies that included restorative care, physical therapy, education and close monitoring.

\subsubsection{Knowledge questionnaire}

The pre- and post-test for knowledge, the Falls Management Program, How to Reduce Falls questionnaire by Agency for Healthcare Quality and Research was used as a self-report questionnaire for participants. ${ }^{[23]}$ Goals of the tests were to identify participant's knowledge of unsafe behaviors that are precursors to falls, as well as specify effective strategies that reduce fall risks. The questionnaire included 5-items (three open ended items; two true-false items). There is no available reliability or validity data available for the questionnaire. The questionnaire is available for use in the public domain by AHRQ, moreover this author received permission to use the questionnaire in this study.

\subsubsection{Staff rounding sheet}

Every two hour rounding with a focus on the 4Ps was instituted with the falls program, and was detailed on every unit on a rounding documentation sheet. Frequency and consistency of staff notated on the rounding sheet on each unit. An effective strategy in this process consists of every two hour rounding with a focus on the 4Ps (potty, pain, positioning, and possession).

\subsubsection{BRIGGS assessment fall risk tool}

The BRIGGS Assessment Fall Risk Tool is an essential component of the fall prevention program, and is utilized to "assess eight parameters of functional status to determine if he resident is at a high risk for falls." [24] The tool is utilized to assess the following risk factors: level of consciousness/mental status, history of falls (past 3 months), ambulation/eliminator status, vision status, gait/balance, systolic 
blood pressure, medications, predisposing disease. On assessment, corresponding scores range from 0 (normal) to 4 (worse/abnormal). A combined score greater than 10 was indicative of high risk for falls. The BRIGGS Assessment fall risk tool is used routinely within the institution for all newly admitted patients; thus, BRIGGS fall risk scores were not documented specifically for this project, but were assessed for the project. When reviewing the evidence, there was no reliability or validity data available.

\subsubsection{Institutional falls data}

The existent quality assurance data was extended through analysis to include: monthly falls rate, calculated as the number of falls/bed-day $\times 1,000$, and severity of injury for each fall, defined as no injury, minor injury (e.g. abrasion, bruise, minor laceration), or major injury (e.g., hip fracture, head trauma, arm fracture). ${ }^{[25]}$ Supplemental quality assurance analysis was evaluated for its effectiveness in program strategies in reducing patient falls.

\subsection{Procedures}

Procedures for the project entailed formalizing a falls risk prevention program, based on informal patterns that were currently utilized within the quality assurance program at the institution. ${ }^{[25]}$ The program included AHRQ recommended parameters for falls prevention. Recruitment of participants and introduction of program objectives with specific verbal instructions were given at the commencement meeting held at the facility. The Principal Investigator (PI) administered The Falls Management Program Pretest. The questionnaire was administered again approximately 2 -months after project implementation, and 4-months post implementation.

\subsection{Analysis}

Dependent variables included: (a) staff knowledge, (b) patient falls risk scores (from Briggs), (c) quality assurance documentation (monthly falls rate, and severity of injury for each fall), (d) documentation of every 2-hr staff rounding documentation, and (e) increased patient activities. All variables were analyzed using descriptive statistics. Trend patterns were documented graphically and the data were disseminated monthly to the Falls Management team. Pre and post-test data were analyzed using descriptive statistics, and statistical analysis was completed using paired $t$-Test comparing pre- and post-test data fall rate at start of implementation to two months and four months post intervention. A $p$-Value of less than .5 was selected to indicate statistical significance with a confidence interval at $95 \%$ for employee knowledge.

The statistical approach used to compare fall rate preimplementation to two months and four months post intervention was the paired-samples $t$ test. Quality assurance data such as number of monthly falls, time of falls, location of falls, resident outcomes, action taken, and physical and occupational therapy services type were observed and summarized although no statistical approach was applied. Final analysis of data was compiled using IBM Statistical Package for the Social Sciences (SPSS) software.

Incidents related to falls were documented by the licensed vocational nurse $(\mathrm{LVN})$ on the Incident/Accident Report and submitted to the DON so the report could be reviewed and discussed within 24 to 72 hours at Morning Meetings. Falls data were regularly collected through the quality assurance department regarding incident and accident data, which included only frequency counts. Pre-implementation, fall data was not routinely shared with staff. Those data was augmented with further analysis regarding fall date and time, location, resident injury, BRIGGS baseline, plan for action, and assessment for needed assistive devices.

Data management and statistical analysis were accomplished using Microsoft Excel 2007 for data compilation, and SPSS version 22.0 .

\section{Results}

\subsection{Knowledge pre- and posttest}

A knowledge test "How to Reduce Falls" was administered to the fall team $(\mathrm{N}=10)$ at baseline, 2-month, and 4-month post-training intervention. The average scores on pre-test, and 2-month and 4-month post-testing were respectively: 74, 90, and 92 (see Table 1).

Table 1. Descriptive Statistics of Pretest, 2-month Post-Test, and 4-month Post-test

\begin{tabular}{lllllll}
\hline Testing & Mean & SD & Min & Max & t-test $(\boldsymbol{d f}=\mathbf{9})^{\#}$ & $\boldsymbol{p}_{\text {-value }}^{\#}$ \\
\hline Pre-test & 74.00 & 18.97 & 40.00 & 18.97 & $* * * *$ & $* * *$ \\
Post-test 2-Mo & 90.00 & 10.54 & 80.00 & 10.54 & $-2.449^{\#}$ & $.037^{\#}$ \\
Post-test 4-Mo & 92.00 & 10.33 & 80.00 & 10.33 & $-2.586^{\#}$ & $.029^{\#}$ \\
\hline
\end{tabular}

Note. Results of ${ }^{\#} t$-test and $p$-values are for the comparison with baseline.

Minimum = lower limit, Maximum = upper limit, $\mathrm{M}=$ mean, $\mathrm{SD}=$ standard deviation, $\mathrm{n}=$ number . 
A paired-samples $t$ test was calculated to compare pretest month $(t(9)=-2.449, p=.037$ and at 4 -month $(t(9)=-2.586$, means to 2- and 4-month means. A significant increase from $p=.029)$ (see Figure 1). baseline to post-testing at two points was demonstrated: at 2-

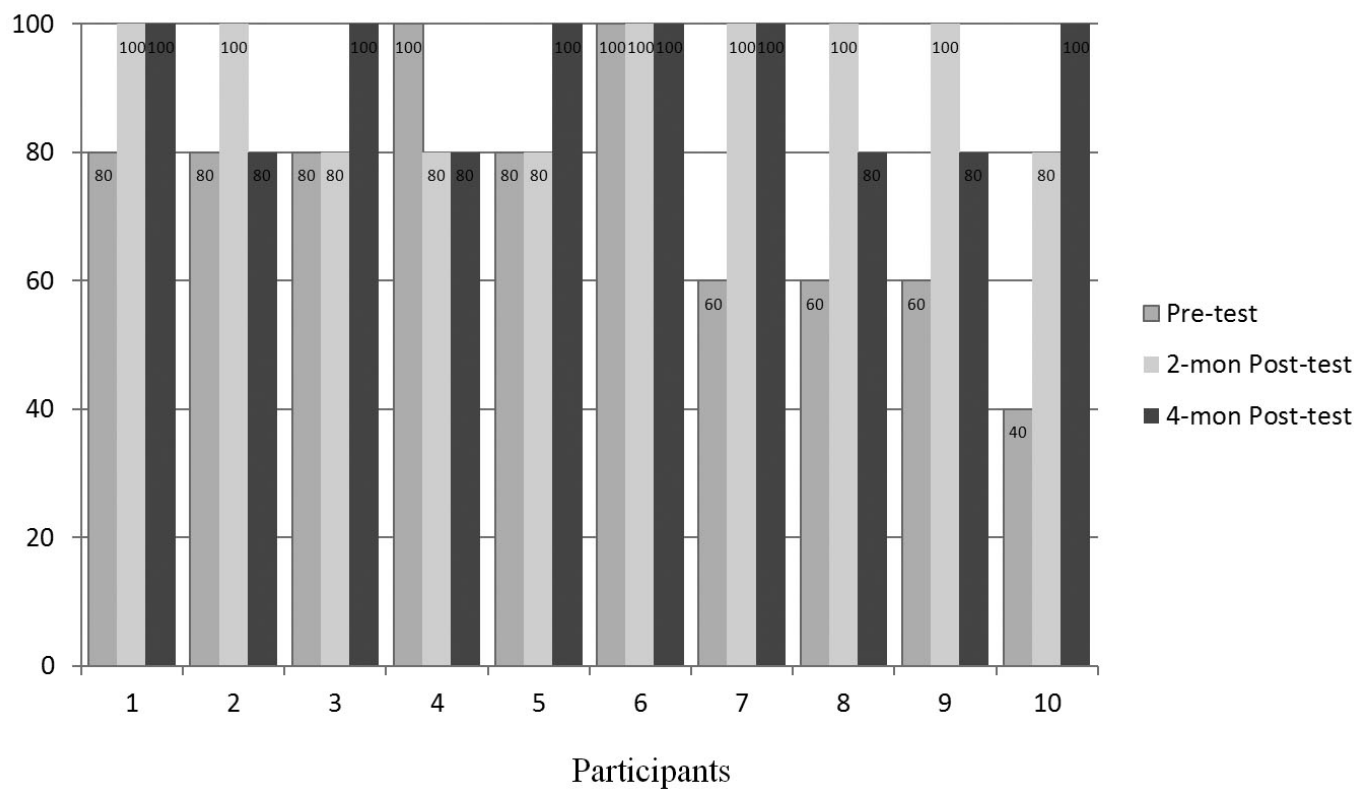

Figure 1. Pre-test, 2-Month and 4-Month Post-tests Scores

\section{2 $\mathrm{Q} 2 \mathrm{H}$ patient rounding}

During the four months of implementation staff completed every two hour rounding for 107 days, documenting on 144 sheets on a total of 454 patients. Combined documentation of the three nursing units was correspondingly: 528/576 entries (91.6\%), 542/576 (94\%), and 516/576 (89.5\%). Average compliance for staff documentation of hourly rounding was $91 \%$.

\subsection{BRIGGS fall risk assessments}

BRIGGS Fall risk assessments were completed for newly admitted patients in July ( $\mathrm{N}=20)$, August $(\mathrm{N}=22)$, September $(\mathrm{N}=18)$, and October $(\mathrm{N}=19)$. The average risk scores for patients during July, August, September, and October were correspondingly: $12.8(\mathrm{SD}=4.15), 10.6(\mathrm{SD}=6.55), 12.4$ $(\mathrm{SD}=4.45)$, and $12.2(\mathrm{SD}=5.18)($ see Table 2$)$.

Table 2. Descriptive Statistics of Pretest, 2-month Post-Test, and 4-month Post-test

\begin{tabular}{lllll}
\hline Assessment Scores & $\boldsymbol{M}$ & $\boldsymbol{S D}$ & $\boldsymbol{L L}$ & $\boldsymbol{U} \boldsymbol{L}$ \\
\hline July & 12.80 & 4.1498 & 7 & 23 \\
August & 10.64 & 6.5505 & 4 & 21 \\
September & 12.39 & 4.4476 & 8 & 20 \\
October & 12.16 & 5.1776 & 10 & 20 \\
\hline
\end{tabular}

Note. $L L=$ lower limit, $U L=$ upper limit, $M=$ mean, $S D=$ standard deviation, $n=$ number.

BRIGGS scores ranged from 4 to 23 and were valuable for assessing risk factors of newly admitted patients (see Table 3 ). Those with scores slightly lower than 10 were typically admitted for short-term therapy. During project implementation $15 \%(n=12)$ of newly admitted had low risk for falls based on their BRIGGS assessments. The predominant assessment scores were between 10 and 15, with 41 (52\%) considered as having high risk for a fall. Nineteen patients (24\%) scored greater than 15 on their BRIGGS assessments. In general, $76 \%$ of patients admitted during the implementation period were determined to have high risk for falls. Increased rates of patients with high risk for falls substantiates the importance of instituting fall management programs that incorporate best practices utilizing multi-interventional strategies for fall prevention in long-term care facilities. ${ }^{[26]}$

\subsection{Average monthly fall rates}

Calculations were completed to determine average monthly fall rates. The average daily census during implementation was as follows: July's census was 114, August was 117, September was 114, and October's census was 108. The average monthly fall rate pre-implementation was 24.5 . Total monthly falls during implementation were in this order: in July there were 13 falls, in August there were 14 falls, in September there were 9 falls, and in October there were 10 falls. Mean scores for monthly falls rate pre-implementation, and 2-month and 4-month post- 
implementation were correspondingly: $24.5(\mathrm{sd}=0.50), 13.5$ $(\mathrm{sd}=0.50)$, and $9.5(\mathrm{sd}=0.50)($ see Table 4$)$. From preimplementation to 2-month post-implementation there was an approximate $45 \%$ reduction in monthly falls. Likewise, from pre-implementation to 4-month post-implementation there was an overall $54 \%$ reduction in average monthly patient falls. The assumption was that fall rates differed significantly indicating post-implementation rates decreased compared to pre-implementation.

Table 3. Frequency of BRIGGS scores by month and by risk category

\begin{tabular}{lllll}
\hline Fall Risk Scores & Low $(<\mathbf{1 0})$ & Moderate (10.1-15) & High $(>\mathbf{1 5})$ & Missing \\
\hline July & 4 & 12 & 4 & 0 \\
August & 6 & 7 & 6 & 3 \\
September & 2 & 11 & 4 & 1 \\
October & 0 & 11 & 5 & 3 \\
\hline
\end{tabular}

Table 4. Descriptive Statistics of Frequency of Falls

\begin{tabular}{llllll}
\hline Average Monthly Falls & M & SD & LL & UL & Months \\
\hline Pre-Implementation & 24.5 & 0.5 & 24.0 & 25.0 & May/Jun \\
2-Month Post-Implementation & 13.5 & 0.5 & 13.0 & 14.0 & Jul/Aug \\
4-Month Post-Implementation & 9.5 & 0.5 & 9.0 & 10.0 & Sep/Oct \\
\hline
\end{tabular}

Note. $L L=$ lower limit, $U L=$ upper limit, $M=$ mean, $S D=$ standard deviation, $n=$ number.

Paired $t$-tests were calculated to compare for falls rate at start of implementation to two months and four months post intervention. Additionally, the paired-samples $t$ test comparing means of pre-implementation to 4-month postimplementation was in this order: $24.5(\mathrm{sd}=0.50)$, and $9.5(\mathrm{sd}=0.50)$. Statistical results indicate there is a significant difference in falls rate comparing pre-implementation to 4-month post-implementation $(\mathrm{t}(2)=25.981, p<.05)$. Evidence suggests that a collaborative care multi-interventional protocol focused on fall prevention improves health outcomes for this population.

\subsection{Fall characteristics - location, timing, outcomes}

Routine data abstraction of fall characteristics was conducted monthly from the Quality Assurance Accident/Incident Report Log. Review of the log allowed retrieval of data regarding average monthly falls, time of fall occurrence (shift), fall location, resident outcomes, and post fall action (see Figure 2). Further breakdown of information during implementation revealed 54 percent of falls occurred during the day, 37 percent during the evening, and 9 percent at night. Equally, 60 percent of patients fell in their rooms, 28 percent in the dayroom, 4 percent in the dining room, and 4 percent in the hallway. There were 42 percent fall related injuries in which 11 percent contributed to fractures, 21 percent skin tears, 16 percent lacerations, and 53 percent bruises/hematomas. Patients received the following post fall actions/interventions:
48 percent care plan revisions, 9 percent therapy initiation, 7 percent hospitalizations, and 6 percent first aid (see Table 5).

Table 5. Frequency of Falls $(N=46)$ Characteristics of falls by Shift, Location at time of fall, Injury status, and Action taken

\begin{tabular}{|c|c|c|c|c|}
\hline$n(\%)$ & $\begin{array}{l}\text { July } \\
(n=13)\end{array}$ & $\begin{array}{l}\text { August } \\
(n=14)\end{array}$ & $\begin{array}{l}\text { September } \\
(n=9)\end{array}$ & $\begin{array}{l}\text { October } \\
(n=10)\end{array}$ \\
\hline \multicolumn{5}{|l|}{ Shift } \\
\hline Day & $8(62)$ & $6(43)$ & $5(56)$ & $6(60)$ \\
\hline Evening & $5(38)$ & $6(43)$ & $2(22)$ & $4(40)$ \\
\hline Night & $0(0)$ & $2(14)$ & $2(22)$ & $0(0)$ \\
\hline \multicolumn{5}{|c|}{ Location at time Fall } \\
\hline Patient Room & $6(46)$ & $11(79)$ & $7(78)$ & $4(40)$ \\
\hline Bathroom & $5(38)$ & $0(0)$ & $1(11)$ & $0(0)$ \\
\hline Dayroom & $1(08)$ & $2(14)$ & $1(11)$ & $5(50)$ \\
\hline Dining Room & $1(08)$ & $0(0)$ & $0(0)$ & $0(0)$ \\
\hline Hall & $0(0)$ & $1(7)$ & $0(0)$ & $1(10)$ \\
\hline \multicolumn{5}{|l|}{ Injury } \\
\hline With & $7(54)$ & $6(43)$ & $1(11)$ & $5(50)$ \\
\hline Without & $6(46)$ & $8(57)$ & $8(89)$ & $5(50)$ \\
\hline \multicolumn{5}{|l|}{ Action } \\
\hline Care Plan Revised & $8(62)$ & $2(14)$ & $7(78)$ & $5(50)$ \\
\hline $\begin{array}{l}\text { Implemented } \\
\text { Therapy }\end{array}$ & 2 (15) & $0(0)$ & $0(0)$ & $2(20)$ \\
\hline Hospitalization & $2(15)$ & $0(0)$ & $0(0)$ & $1(10)$ \\
\hline First Aid & $1(08)$ & $0(0)$ & $0(0)$ & $2(20)$ \\
\hline
\end{tabular}




\section{Discussion}

Overall, results of the project does support the proposed hypothesis that collaborative care multi-interventional fall prevention interventions reduce risk and prevalence of patient falls in LTC facilities. Implementation of the project examined the efficacy of a multi-interventional fall prevention program designed for reducing elderly patient falls in the nursing home. There was a 20 percent increase in participant's knowledge retention demonstrated between 2-month and 4 month implementation periods. During the four months of implementation staff completed every two hour rounding and documentation 91 percent of the time. There was a significant difference in monthly fall rate over a 4-month period in which fall rates decreased by 53 percent comparing baseline to post implementation periods.

The goal of the capstone project was to complement fall prevention guidelines addressed by The Joint Commission's ninth goal, which strategically endorses reducing the risk of patient harm resulting from falls. Findings from this study can be compared to positive outcomes of studies in the literature, such as Jung and Lee (2009) in which their study demonstrated there was a positive correlation regarding combined exercise and education. ${ }^{[27]}$ Similarly, findings of this study can be compared to results of the quasi-experimental study which established a reduction in patient falls clinically, but lacked significance statistically (Olrich, Kalman, \& Nigolian, 2008). ${ }^{[28]}$

\subsection{Implications for practice}

Implementing the program allowed frontline caregivers to maximize care delivery utilizing evidence-based practices associated with reducing barriers and utilizing effective interventions that improved patient fall rates. The Joint Commission acknowledged its concern for falls assessment and prevention since 2005, and continues to list it as one of its national safety standards focusing on prevention $(2007,2008)$. Integration of the multi-interventional protocol focuses on a fall prevention program that fosters joint responsibility between nursing leaders and staff to demonstrate competencies, skills and resources necessary for providing quality, safe care that effect desired outcome of decreasing fall rates in the elderly. It is anticipated that information gained from results of this project could be used to foster continued quality improvement methods that facilitate ongoing clinical practices to augment health and wellness experiences of patients, and improve quality of care.

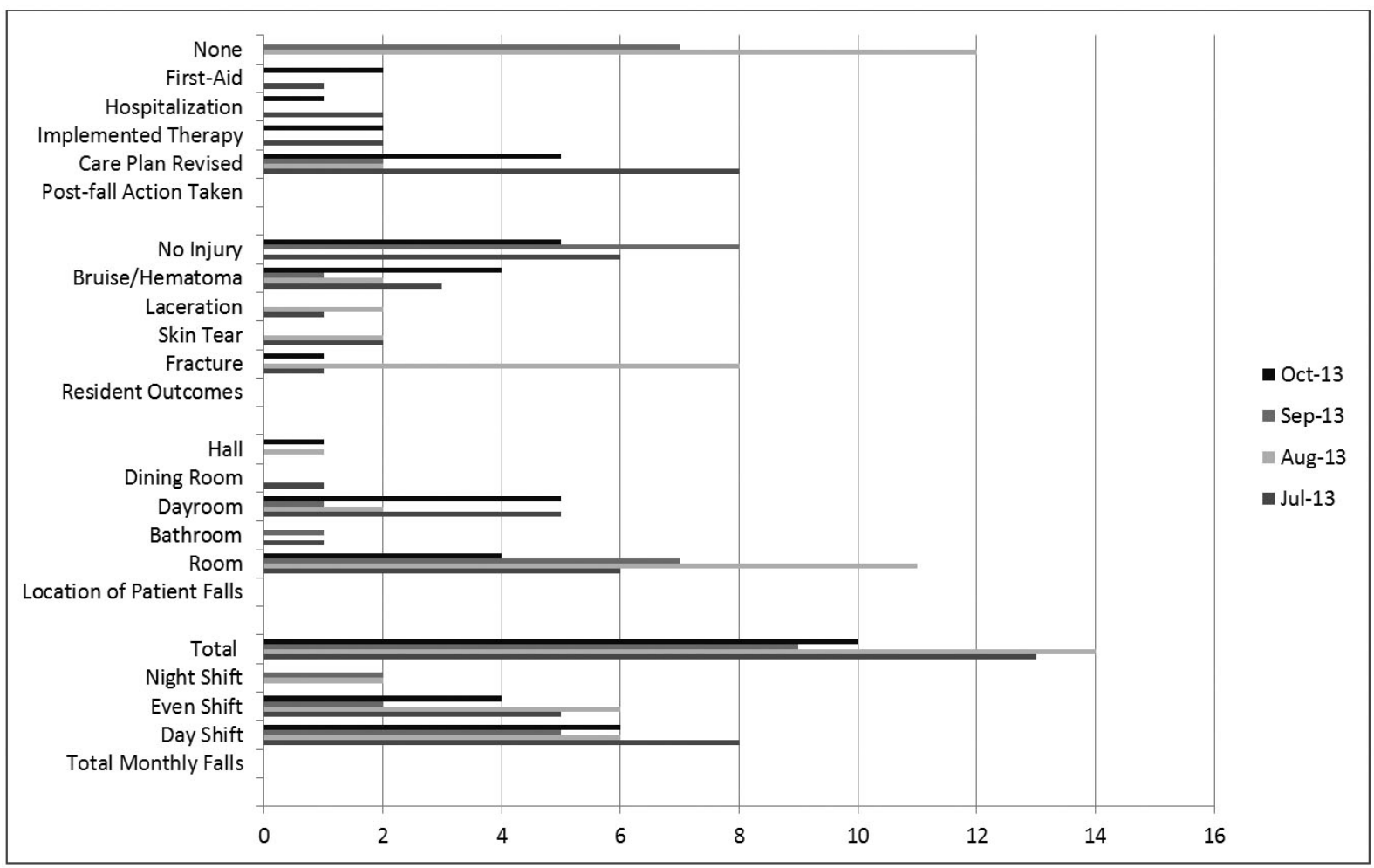

Figure 2. Bar graph displaying quality assurance patient falls data for selected months 
Implications for future studies require support of fall prevention and collaborative measures that promote education and training, enforce adherence to appropriate standards of care, and opportunities for continued research. Encouraging an environment that promotes cooperative engagement in education and training between staff, patients and their families, in addition to developing, implementing evidence-based protocols related to falls management may achieve positive outcomes. Healthcare clinicians who proactively collaborate and engage patients in the delivery of care provide effective, efficient and timely care. ${ }^{[29]}$ It is equally imperative for patient advocates to pursue advancement of stringent state-wide policies which survey and control fall prevention standards and compliance in long term care facilities. The importance of understanding and reducing potential risks of falls means it is essential that clinicians continue research efforts to establish strategies for fall prevention that create positive outcomes in the best interest of the elderly under our care. The prevalence of patient falls and fall-related injuries continue to challenge healthcare providers, therefore we must do diligence in researching opportunities that foster employing effective strategies for screening, implementing, and measuring patient safety in fall prevention.

\subsection{Project limitations}

One of the limitations associated with this quantitative project was the small sample size. Having a small sample size may increase risks for statistical error, and also affect validity of findings. ${ }^{[30]}$ Noteworthy regarding a small sample size is that even if there is no statistical difference, there may be a significant clinical difference. Consequently, for the project there was no identified impact on data collection, analysis, or overall project findings. Because the facility is one of 150 nursing homes under a large corporate structure, future plans include presenting the project to corporate leaders and taking advantage of implementing it at other sites which may show similarities of results in the different settings.

A procedural limitation of the project entailed the rounding approach. The every 2-hour rounding notation sheet required modification after the project began. It was identified that having the rounding notation sheets on every patient became too time consuming for compliance with documentation given that units could be bedded to capacity with 40 to 50 patients. Furthermore, having individual sheets for each patient would require too much paper in charts (30 sheets per month). The process was effectively and efficiently modified, and it improved compliance once individual 24-hour sheets were placed in binders on each of the three nursing units. Documentation was completed every two hours for the entire unit; however the limitation of this approach was that one couldn't evaluate if each patient had been rounded on.

\subsection{Dissemination and sustainability}

According to Lloyd (2011), ${ }^{[31]}$ demonstrated falls tracking is a significant undertaking for nursing which may provide necessary information related to the success or failure of any existing or future interventions, may necessitate improvements be made, and facilitate information sharing. The demonstration of positive results, such as decreased fall rates was instrumental for sustaining the project on a long-term basis. It necessitates committing to continual organizational auditing of internal processes to ensure achievement of performance improvement related to national and local standards of excellence. As well, the project promotes opportunities to correct deficiencies, provide high quality care to patients in the most efficient and cost effective manner, and maintain patient and employee satisfaction.

Introducing findings from the capstone project will afford opportunities to expand the program with ongoing education and training for care providers advancing standards of care in fall prevention. Project results will be reported to organizational leaders and stakeholders with the intent of having it implemented at every one of their 190 skilled nursing home affiliates. Ongoing collaboration with organizational leaders will be focused on effectively improving work performance and patient safety, as well as developing new or revising current policies related to reducing patient falls and improving patient outcomes. In addition, my objective includes sharing of information by means of educating and empowering healthcare providers and advocates by presenting at health care conferences on the utilization of best practices in fall prevention, expressly effectiveness of capstone initiatives and outcomes.

In regards to additional future research initiatives, the PI will explore opportunities that contribute to the body of nursing knowledge by publishing project findings in scholarly nursing journals. Moreover, the PI will aspire to collaborate and build partnerships with key healthcare practitioners, community and political leaders to advocate for state-wide policies that mandate higher standards of care for nursing homes to comply with developing and implementing methods to improve patient safety, and supporting clinical functions and processes with best practices.

\subsection{Review of literature}

A literature search was conducted using CINAHL, Medline, Ovid, and Google Advance Scholar. Key words used in the searches included patient falls, fall prevention, elderly falls, geriatric safety, fall prevention strategies, and nursing home 
falls. Review of articles included dates between 2006 to current, and restricted to English language. The literature demonstrates that there are successful fall prevention strategies, and a multifaceted fall management program inclusive of an interdisciplinary approach is effective for reducing the incidence of falls in the elderly.

According to the AHRQ, approximately 700,000 to $1,000,000$ people in the United States experience falls and fall-related injuries in hospitals that result in fractures, lacerations, internal bleeding, and increased health care utilization. ${ }^{[22]}$ Additional consequences of falls include delayed healing, pain and suffering, fear of future falls, and mistrust in caregivers, all of which impact patient's quality of life. And, researchers have reported that nearly one-third of patient falls can be prevented. Furthermore, AHRQ has developed a toolkit specifically for improving quality of care and reducing falls by means of developing, implementing, and sustaining an interdisciplinary approach to care.

Other interventions in the literature focused on determining the effects of exercise on falls prevention in patients 65 years and older include establishing whether particular trial characteristics or components of exercise programs are associated with larger reductions in falls, and research to determine the effects of exercise on falls prevention in older people understanding whether particular trial characteristics or components of exercise programs are associated with larger reductions in falls. ${ }^{[32]}$ The investigation measured exercise, muscle strength, gait, balance training, and strength training and its effect on fall rates. Findings are consistent with prior protocols and indicate a key feature regarding the significance of designing and implemented exercise programs can be effective in fall prevention.

Anderson et al. (2012) $)^{[33]}$ conducted a RCT to assess the effectiveness of interventions designed to prevent patient injuries and falls from their beds. The sample included 22,106 participants. Variables studied were bed rails, low height beds, bed exit alarms, bed-side floor mats, and an educational program. Researchers used rate ratios as the statistical test for measurement. Upon completion of the study, the results concluded no significant increase or decrease in rate of injuries or falls from bed. Weakness of the study indicated a lack in blending of staff and insufficient power, no RCT of bed rails were identified, and it lacked high quality evidence for/or use of intervention. Further research was recommended.

In the literature, a quantitative systematic review was conducted to assess the effectiveness and characteristics of fall prevention interventions implemented in hospitals, which facilitate the development of a multi-systemic fall prevention model that established a practical framework. ${ }^{[34]}$ This study included review of 34 studies. Variables examined included the physical environment, care process and culture, and technology. A $t$-test was used, and upon completion of the study results revealed development and implementation of a multi-systemic fall prevention model that incorporates the physical environment, the care process and technology effectively prevents falls, and is beneficial to the well-being of patients and caregivers. Weaknesses of the study included lack of pooled results, inability to identify total sample size, and the potential risk of bias.

A meta-analysis of RCTs was conducted to assess the effects of interventions designed to reduce the incidence of falls in older people in care facilities and hospitals. ${ }^{[27]}$ The study included 60 trials of which 60,345 were participants. Variables studied were exercise, vitamin D supplements, and floor carpeting. The statistical test used was rate ratio at 95\% confidence interval (CI). Upon completion of the study, the results illustrated exercise interventions were inconsistent between intervention and control groups in reducing falls, vitamin D supplements reduced falls, but not risk of falling, and multidisciplinary care after hip fracture surgery compared to usual care reduced rate of falls. Weaknesses included limited evidence supporting outcomes.

A clustered RCT was performed to evaluate the benefits and harms of rehabilitation interventions directed at maintaining or improving, physical function for older-people in long-term care through the review of randomized and cluster randomized control trials. ${ }^{[35]}$ Researchers studied 67 trials with a total of 6,300 participants. Study variables were exercise tolerance strength, flexibility, balance, and perceived health status. Statistical test used was the rate ratio with $95 \%$ CI. Upon completion of the study, researchers indicated rehabilitation treatment may be effective in improving the physical health of older people in long-term care. Weakness of study noted possible bias may have resulted in overestimation of the positive effects of physical rehabilitation.

\section{ACKNOWLEDGEMENTS}

This author thanks Dr. Patricia Pearce (Chair Advisor) for sharing wisdom, support and guidance throughout the capstone process, and Dr. Shannan Hamlin for her invaluable influence in understanding the importance of disseminating this study's findings.

\section{CONFlicts OF InTEREST Disclosure}

The author declares that there is no conflict of interest statement. 


\section{REFERENCES}

[1] Kannus P, Parkkari J, Niemi S, et al. Fall-induced deaths among elderly people. American Journal of Public Health. 2005; 95(3): 422-424. http://dx.doi .org/10.2105/AJPH. 2004.047779

[2] Centers for Disease Control and Prevention, National Center for Health Statistics. 2011. Residential Care Facilities: A Key Sector in the Spectrum of Long-term Care Providers in the United States. Available from: http://www.cdc.gov/nchs/data/databriefs $/ \mathrm{db} 78 . \mathrm{htm}$

[3] Rapp K, Lamb SE, Bucele G, et al. Prevention of falls in nursing homes: subgroup analyses of a randomized fall prevention trial. Journal of American Geriatric Society. 2008; 56: 1092-1097. http://dx.doi.org/10.1111/j.1532-5415.2008.01739.x

[4] Sherrington C, Whitney JC, Lord SR, et al. Effective exercise for the prevention of falls: A systematic review and meta- analysis. Journal of American Geriatric Society. 2008; 56: 2234-2243. PMid:19093923 http://dx.doi.org/10.1111/j.1532-5415.2008.02014.x

[5] Anderson O, Boshier PR, Hanna GB. Interventions designed to prevent healthcare bed-related injuries in patients (Review). The Cochrane Collaboration. Published by JohnWiley \& Sons, Ltd. 2012.

[6] Spiva L, Robertson B, Delk ML, et al. Effectiveness of team training on fall prevention. Journal of Nursing Care Quality. 2014; 29(2): 164173. http://dx.doi.org/10.1097/NCQ . 0b013e3182a98247

[7] Oliver D, Connelly JB, Victor CR, et al. Strategies to prevent falls and fractures in hospitals and care homes and effect of cognitive impairment: systematic review and meta-analyses. British Medical Journal. 2007; 334(7584): 82-85. http://dx . doi .org/10.1136 /bmj.39049.706493.55

[8] Rubenstein LZ. Falls in older people: Epidemiology, risk factors and strategies for prevention. Age Ageing. 2006; 35(Suppl. 2): ii37-ii41. http://dx.doi.org/10.1093/ageing/af1084

[9] Centers for Disease Control and Prevention, National Center for Injury Prevention and Control. Web-based Injury Statistics Query and Reporting System (WISQARS). Available from: http://www.cdc.gov/homeandrecreationalsafety/ falls/adultfalls.html

[10] National Database of Nursing Quality Indications. Guidelines for data collection on the American Nurses Association's national quality forum endorsed measures: Nursing care hours per patient day skill mix falls with injury. 2010. Available from: https://www. nursin gquality.org/data.aspx

[11] Bergmann TF. Taking on the fall: The etiology and prevention of falls in the elderly. Clinical Chiropractic. 2008; 11(3): 148-154. http://dx.doi.org/10.1016/j.clch.2008.06.001

[12] Hill EE, Nguyen TH, Shaha M, et al. Person-environment interactions contributing to nursing home resident falls. Research in Gerontological Nursing. 2009; 2(4): 287-296. PMid:20077985. http://dx.doi.org/10.3928/19404921-20090527-02

[13] Chou KL, Yeung FK, Wong EC. Fear of falling and depressive symptoms in Chinese elderly living in nursing homes fall efficacy and activity level as mediator or moderator? Aging Mental Health. 2005; 9(3): 255-261. PMid:16019279. http://dx.doi.org/10.1080/1 3607860500114035

[14] Peel NM. Epidemiology of falls in older age. Canadian Journal of Aging. 2011; 15: 1-13.

[15] Lach HW, Ball LJ, Birge SJ. The nursing home falls self-efficacy scale: Development and testing. Clinical Nursing Research. 2012; 21(1): 79-91. http://dx.doi.org/10.1177/1054773811426 927

[16] Stevens JA, Sogolow ED. Gender differences for non-fatal unintentional fall related injuries among older adults. Injury Prevention.
2005; 11: 115-119. http://dx.doi.org/10.1136/ip.2004.0 05835

[17] Rapp K, Lamb SE, Bucele G, et al. Prevention of falls in nursing homes: subgroup analyses of a randomized fall prevention trial. Journal of American Geriatric Society. 2008; 56: 1092-1097. http://dx.doi.org/10.1111/j.1532-5415.2008.01739.x

[18] Williams T, Szekendi M, Thomas S. An analysis of patient falls and fall prevention programs across academic medical centers. Journal of Nursing Care Quality. 2014; 29(1): 19-29. PMid:23842442 http://dx.doi.org/10.1097/NCQ.0b013e3182a0cd19

[19] McCormack B, McCance TV. Development of a framework for person-centered nursing. Journal of Advanced Nursing. 2006; 56: 472-479. PMid:17078823 http://dx.doi.org/10.1111/j.136 $5-2648.2006 .04042 \cdot x$

[20] Tzeng H. Nurses' caring attitude: Fall prevention program implementation as an example of its importance. Nursing Forum. 2011; 46(3): 137-145. PMid:21806622 http://dx.doi.org/10.1111/j.174 4-6198.2011.00222.x

[21] Cozart HCT, Cesario SK. Falls aren't us: State of the science. Critical Care Nursing Quarterly. 2009; 32(2): 116-127. PMid:19300076. http://dx.doi.org/10.1097/CNQ.0b013e3181a27dc0

[22] Agency for Healthcare Research \& Quality. Preventing Falls in Hospitals: A Toolkit for Improving Quality of Care (AHRQ Publication No. 13-0015-EF). 2013. Available from: http://www. ahrq.gov/leg acy/research/ltc/fallpxtoolkit/fallpxtoolkit.pdf

[23] Agency for Healthcare Research \& Quality. The Falls Management Program: A Quality Improvement Initiative for Nursing Facilities (AHRQ Publication No. 290-00-0011 Task Order No. 3). 2005 Available from: http://www.ahrq.gov/sites/default/fil es/wysiwyg/professionals/systems/long-term-care/re sources/injuries/fallspx/fallspxmanual.pdf

[24] BRIGGS Healthcare. Fall Risk Assessment. Briggs Corporation. 2003. Available from: https://shop.briggscorp.com/e2wRec entlyViewedALLItems . aspx

[25] Quality Assessment and Assurance Committee Record (2011, May 31). Quality of care, 42 U.S.C. $\S \S 1395 i-3(b)(1)(B)$ and 42 CFR $\S$ 483.75(0).

[26] Rask K, Parmelee PA, Taylor JA. Implementation and evaluation of a nursing home fall management program. Journal of American Geriatric Society. 2007; 55: 342-349. PMid:17341235 http: //dx.doi.org/10.1111/j.1532-5415.2007.01083.x

[27] Jung D, Lee J, Lee S. A meta-analysis of fear of falling treatment programs for the elderly. Western Journal of Nursing Research. 2009; 31: 6-16. Available from: http://wjn. sagepub.com/content/ $31 / 1 / 6$

[28] Olrich T, Kalman M, Nigolian C. Hourly rounding: a replication study. Medsurg Nursing. 2008; 21(1): 23-26.

[29] Merrett A, Thomas P, Stephens A, et al. A collaborative approach to fall prevention. Canadian-Nurse.Com. 2011; 107(8): 24-29.

[30] Melnyk BM, Fineout-overholt E. Evidenced-Based Practice in Nursing \& Healthcare: A Guide to Best Practice. Philadelphia, PA: Wolters Kluwer/Lippincott Williams \& Wilkins. 2001.

[31] Lloyd T. Creation of a multi-interventional fall prevention program: Using evidence-based practice to identify high-risk units and tailor interventions. Orthopedic Nursing. 2011; 30(4): 249256. PMid:21799380 http://dx.doi .org/10.1097/NOR.0b013 e3182247c61

[32] Sherrington C, Whitney JC, Lord SR, et al. Effective exercise for the prevention of falls: A systematic review and meta-analysis. Journal of American Geriatric Society. 2008; 56: 2234-2243. PMid:19093923 http://dx.doi.org/10.1111/j.1532-5415.2008.02014.x 
[33] Anderson O, Boshier PR, Hanna GB. Interventions designed to prevent healthcare bed-related injuries in patients (Review). The Cochrane Collaboration. Published by JohnWiley \& Sons, Ltd. 2012.

[34] Choi Y, Lawler E, Boenecke CA, et al. Developing a multi-systemic fall prevention model, incorporating the physical environment, the care process and technology: A systematic review. Journal of
Advanced Nursing. 2011; 67 (12): 2501-2524. PMid:21545639 http://dx.doi.org/10.1111/j.1365-2648.2011.05672.x

[35] Crocker T, Forster A, Young J, et al. Physical rehabilitation for older people in long-term care. Cochrane Database of Systematic Reviews. 2013; (2): 1-182. 